\title{
PACIFIC: shifting tides in the treatment of locally advanced non-small cell lung cancer
}

\author{
Andrew Bang ${ }^{1}$, Jonathan D. Schoenfeld ${ }^{1}$, Alexander Y. Sun ${ }^{2}$ \\ ${ }^{1}$ Department of Radiation Oncology, Brigham and Women's Hospital/Dana-Farber Cancer Institute, Boston, MA, USA; ${ }^{2}$ Department of Radiation \\ Oncology, Princess Margaret Cancer Centre/University of Toronto, Toronto, ON, Canada \\ Contributions: (I) Conception and design: All authors; (II) Administrative support: None; (III) Provision of study materials or patients: None; (IV) \\ Collection and assembly of data: A Bang; (V) Data analysis and interpretation: All authors; (VI) Manuscript writing: All authors; (VII) Final approval \\ of manuscript: All authors. \\ Correspondence to: Alexander Y. Sun, MD, Associate Professor. Department of Radiation Oncology, Princess Margaret Cancer Centre/University of \\ Toronto, 610 University Ave, Toronto, ON M5G 2M9, Canada. Email: alex.sun@rmp.uhn.ca.
}

\begin{abstract}
The treatment paradigm of stage III, unresectable non-small cell lung cancer (NSCLC) has had few advancement since concurrent chemoradiotherapy was established as standard of care treatment. Despite modifications to radiotherapy, chemotherapy and surgical approaches, loco-regional and distant relapse remain high, which unfortunately has translated to poor survival outcomes. The PACIFIC study introduced immunotherapy to the domain of stage III NSCLC and has emerged as the fourth pillar in cancer treatment for these patients. The positive results of the study have excited both the radiation and medical oncology communities, demonstrating improvements in overall and progression-free survival (PFS). In this review, we discuss the details and impacts of the PACIFIC study, as well as the future implications for the treatment of stage III NSCLC.
\end{abstract}

Keywords: PACIFIC; immunotherapy; chemoradiation; non-small cell lung cancer (NSCLC)

Submitted Apr 10, 2019. Accepted for publication Aug 29, 2019.

doi: $10.21037 /$ tlcr.2019.09.04

View this article at: http://dx.doi.org/10.21037/tlcr.2019.09.04

\section{Background}

Lung cancer has the highest incidence and mortality rates of all cancers worldwide, representing $11.6 \%$ and $18.4 \%$ of all cancer diagnoses and causes of cancer death (1). The majority of lung cancer patients are diagnosed with non-small cell disease, with $35 \%$ of these patients under the subset of locally advanced non-small cell lung cancer (LA-NSCLC) (2). In cases of unresectable stage III cancer, concurrent chemoradiation is the standard of care with 5 -year overall survival (OS) rates between $15-32 \%(2,3)$. Although newer studies appear to show encouraging improvements in survival, this is likely largely driven by stage migration with increasing use of ${ }^{18} \mathrm{~F}-\mathrm{FDG}$ PETCT and MRI brain at the time of initial diagnosis (4). Nevertheless, survival outcomes are still poor.

With nearly $40 \%$ of patients experiencing a local recurrence (5), efforts have focused on enhancing standard concurrent chemoradiation to improve local control, which could perhaps circumvent distant spread, and thus increase subsequent survival. Improvements in radiotherapy delivery systems and image guidance have enabled the exploration of escalating total radiation dose. RTOG 0617 randomized patients with unresectable stage III NSCLC to receive a standard dose of 60 or Gray (Gy) in the experimental arm with a primary endpoint of OS (5). The investigators concluded that dose escalation was not superior, and even potentially harmful, as the median OS was 20.3 months in the experimental arm compared to 28.7 months in the standard dose arm (5). The reasons behind the survival detriment are still unclear and debated, however increased cardiac radiation dose was considered as a significant contributor to overall mortality (6).

The improved availability of imaging modalities such 
as ${ }^{18} \mathrm{~F}$-FDG PET-CT has allowed for more selective doseescalation with the goal of improving loco-regional control without worsening acute and long-term toxicities. There are currently three randomized phase II studies exploring this question utilizing ${ }^{18} \mathrm{~F}-\mathrm{FDG}$ PET-CT in the treatment planning process to determine which volumes to boost: RTOG 1106 (NCT01507428), NKI/European study (NCT01024829) and the Canadian study (NCT02788461). It should be noted that the Canadian study, which was developed more recently, is accruing patients who receive consolidative durvalumab as part of their standard of care treatment (7). Kong et al. published the outcomes of their single-institution trial, which used a mid-treatment integrated PET-boost, with a median OS of 25 months that is consistent with historical outcomes (8). However, the 2-year loco-regional control was $62 \%(8)$, which is encouraging as we await the results of the three randomized studies.

In addition to modifying radiotherapy parameters, the use of consolidative chemotherapy following concurrent chemoradiation has been investigated. In a randomized phase III study by Ahn et al., patients were randomized between chemoradiation (66 Gy in 33 fractions with weekly cisplatin/docetaxel) and the same chemoradiation with the addition of three cycles of consolidative cisplatin/docetaxel given every three weeks (9). There was no improvement in progression-free survival (PFS) or OS in the experimental arm (9). Different chemotherapy regimens were also explored, specifically in the PROCLAIM study, which investigated pemetrexed for its radiosensitizing properties. Patients were randomized to receive a combination of cisplatin/etoposide in the standard arm or cisplatin/ pemetrexed in the experimental arm, and both arms received consolidative chemotherapy of their respective drugs (10). Although there did appear to be improvements in the toxicity profile with pemetrexed, it was not superior to etoposide in combination with cisplatin with regards to OS (10). Despite the efforts of both the radiation and medical oncology community, it appeared that traditional chemoradiation had reached a plateau with regards to its effectiveness in treating LA-NSCLC, paving the way for a necessary shift in the treatment paradigm.

Immunotherapy has generated significant interest in the treatment of NSCLC, with the recent success of immune checkpoint inhibitors (ICI) in the metastatic, stage IV setting. Blockade of programmed-cell death 1 (PD-1) has been demonstrated to have higher response rates and improved survival compared to second-line chemotherapy
(11-13), and first-line chemotherapy (14) for patients with high programmed death-ligand 1 (PD-L1) expression (tumor proportion score $\geq 50 \%$ ). PD-L1 inhibitors have also shown clinical benefit with atezolizumab demonstrating superior OS to second-line docetaxel $(15,16)$. In addition to $\mathrm{PD}-1 / \mathrm{PD}-\mathrm{L} 1$ inhibitor monotherapy, combination therapies have been investigated. The addition of the cytotoxic T-lymphocyte-associated protein 4 (CTLA-4) inhibitor ipilimumab has been shown to increase the objective response rate (17). Similarly, adding cytotoxic chemotherapy appears to synergize with blockade of the PD-1/PD-L1 axis (18).

Given the positive outcomes of combination therapy with PD-1 inhibitors, radiotherapy has also emerged as a candidate for investigating potential synergistic benefits. The interaction between the effectiveness of radiotherapy and a functioning immune system is well documented. In the pre-clinical setting, it has been demonstrated that blockade of cytotoxic T-cell function is correlated with increased tumor volume growth and poorer survival with equivalent radiotherapy treatments (19). The mechanisms of cellular damage and death induced by focused radiotherapy can enhance anti-tumor immune responses (20). Although large field radiation has historically been appreciated for its immunosuppressive ability (21), targeted radiotherapy can induce substantial changes in the tumor microenvironment that induce innate and adaptive immune responses. The immunogenic effects of radiation include enhanced STING pathway activation, antigen presentation, T-cell activation and trafficking that may complement the immune effects of both CTLA-4 and PD-1 pathway inhibition (22). These interactions may also be dependent on the timing of treatment, as murine model data have shown improved outcomes when PD-1/PD-L1 inhibitors were administered during, as opposed to after radiotherapy (23). Preclinical studies have also demonstrated synergies to improve both local and distant control, including abscopal regression of established tumors outside of the radiation treatment field (24). However, there is limited clinical data that demonstrates substantial improvements in outcomes with the addition of ICI to radiotherapy.

\section{PACIFIC study}

Several landmark studies demonstrated the effectiveness of the PD-1 inhibitors pembrolizumab and nivolumab in the first and second-line setting for stage IV NSCLC. Durvalumab (MEDI4736) is a monoclonal antibody, which 
also inhibits the PD-1/PD-L1 axis by binding PD-L1 (25). It was investigated in several studies for advanced NSCLC including the ATLANTIC study as monotherapy, and the MYSTIC trial in combination with the CTLA-4 inhibitor tremelimumab. The ATLANTIC study was a phase II, single-arm study in the third-line setting, for patients with stage IIIB or IV disease. The study demonstrated objective response rates of $7.5 \%, 16.4 \%$ and $30.9 \%$ with PD-L1 expression levels of less than $25 \%$, between $25 \%$ and $90 \%$ and greater than $90 \%$ respectively (26). In the context of the heavily pretreated nature of the patient cohort, the authors concluded that regardless of PD-L1 expression status, durable responses were achieved.

These findings led to the development of the PACIFIC study, which was the first trial to add immunotherapy to enhance curative treatment for LA-NSCLC. It was designed as a multicenter randomized study with histologically or cytologically documented stage III, unresectable NSCLC by the $7^{\text {th }}$ edition TNM staging system. Patients were randomized within 1 to 42 days of completing concurrent chemoradiation in a 2:1 ratio to receive durvalumab or placebo every two weeks for a maximum duration of 12 months (27). Patients were stratified by age, sex and smoking history (27). The treatment parameters of chemoradiation were heterogeneous, with a variety of platinum-based doublet chemotherapies available, and the radiation dose ranging from 54 to 66 Gy (27). Several key exclusion criteria included previous exposure to PD-1/PDL1 inhibitors, history of autoimmune disease or primary immunodeficiency and grade $2+$ pneumonitis from previous chemoradiation (27). The co-primary endpoints for the study were PFS and OS, both defined from the time of randomization. Secondary endpoints included objective response rate, duration of response, time to distant metastases or death, and safety profile. Disease responses were assessed by a blinded central review using RECIST v1.1 and toxicity was graded using CTCAE v4.03 (27).

A total of 713 patients were randomized, of which 709 received at least one dose of the study drug. The majority of patients enrolled in the study were men $(70 \%)$ and had a history of smoking (91\%) (27). The stage breakdown was balanced between stage IIIA (52.9\%) and stage IIIB (44.7\%), as was tumor histology between squamous $(45.7 \%)$ and nonsquamous (54.3\%) types (27). A large proportion of patients had a partial response $(48.1 \%)$ or stable disease $(47.1 \%)$ following chemoradiotherapy (27). The consolidative durvalumab and placebo arms were balanced between the two groups by baseline and treatment characteristics. A planned interim analysis for PFS was performed, leading to the initial publication in 2017; mature data including OS outcomes were presented at the World Conference of Lung Cancer (WCLC) 2018 and published shortly thereafter.

\section{Survival outcomes}

\section{PFS and distant metastasis-free survival}

In the initial publication, the co-primary endpoint of PFS was determined to be significantly improved with the addition of consolidative durvalumab. In the updated publication in 2018, the median PFS was found to be 17.2 months with durvalumab compared to 5.6 months with placebo; the hazard ratio was 0.51 (95\% CI: 0.41-0.63, $\mathrm{P}<0.001)(28)$. The 12 - and 18 -month PFS rates were $55.9 \%$ vs. $35.3 \%$ and $44.2 \%$ vs. $27.0 \%$ in the two arms respectively, with clear improvements when durvalumab was added (27). Also of note was the improvement in the time to death or distant metastasis: 28.3 months compared to 16.2 months and a hazard ratio of 0.53 (95\% CI: 0.41-0.68). In addition, patients had lower rates of developing brain metastases (6.3\% vs. 11.8\%) (28), which could be the result of blood-brain barrier penetration of the drug, treating pre-existing micrometastases, or possibly a reduction in metastatic seeding due to better overall control of the disease burden. These impressive PFS outcomes led to the Food and Drug Administration (FDA) approval of durvalumab in February 2018 and Health Canada approval in May 2018.

\section{OS}

With the significant improvements in PFS, there was plenty of enthusiasm in the oncology community that these results would translate to a benefit in OS. Given the durable responses stemming from augmentation of the adaptive immune system seen with ICIs in metastatic NSCLC and other histologies, the excitement appeared justified. Almost one year after the initial publication, the updated analysis was published validating this belief. Consolidative durvalumab improved OS with a hazard ratio of 0.68 (99.73\% CI: $0.47-0.997 ; \mathrm{P}=0.0025)$ and the 1 - and 2-year OS rates for durvalumab compared to placebo were $83.1 \%$ vs. $75.3 \%$ and $66.3 \%$ vs. $55.6 \%$ respectively (28). Borne out by these figures, it was also reassuring to note that the improvements in survival were sustained beyond 2 years, although this would need to be confirmed by future long-term analysis. 


\section{Sub-group analyses}

Overall, both publications state that the PFS and OS benefits were observed across the pre-specified subgroups $(27,28)$. In the initial publication, aside from EGFR mutation status, all subgroups appeared to have improved PFS with durvalumab compared to placebo. This included PD-L1 expression, with hazard ratios of 0.41 (95\% CI: $0.26-0.65)$ and 0.59 (95\% CI: 0.43-0.82) for patients with greater and less than $25 \%$ expression levels respectively (27). However, the subgroup analysis in the updated publication for OS showed interesting trends, which could prompt further investigation for optimizing patient selection. Certainly, any observations are not conclusive as the study was not powered for these subgroup analyses (29).

With regards to OS, several subgroup analyses of interest included sex, region, PD-L1 expression status, and time from last radiation treatment to randomization. There were fewer female patients enrolled on the study, representing $30 \%$ of patients, but they had an improved hazard ratio of 0.46 (95\% CI: $0.30-0.73$ ) compared to male patients with a hazard ratio of 0.78 (95\% CI: 0.59-1.03) (28). However, it should be noted that in a meta-analysis of 23 randomized trials in solid tumors, sex did not appear to affect efficacy of immunotherapy (30). Patients from the Americas (HR 0.46) appeared to have better outcomes with durvalumab in comparison to patients accrued in European (HR 0.86) and Asian (HR 0.67) countries (28).

The hazard ratios for patients with $\geq 25 \%$ PD-L1 expression and $<25 \%$ were 0.46 (95\% CI: $0.27-0.78$ ) and $0.92(0.63-1.34)$, respectively (28). This contrasts with the subgroup analysis seen for PFS in the interim analysis. Looking carefully at the supplementary materials, it appears that this discrepancy may be largely driven by the patient cohort with $<1 \%$ PD-L1 expression which had a hazard ratio of 1.36 for OS when given durvalumab (28). However, as cautioned before, the study was not powered to determine efficacy in these subpopulations, particularly when subsequent treatments following durvalumab may have been heterogenous. Finally, the subgroup analysis examining the timing of durvalumab administration, using time to randomization from radiotherapy as a surrogate, demonstrated increased effects when randomization occurred within 14 days as opposed to after, with hazard ratios of 0.42 (95\% CI: $0.27-0.67)$ and 0.81 (95\% CI: 0.62-1.06) (28). This could indicate that the temporal proximity of treatments may be an important factor in optimizing the combination of these therapies. However, this result is also confounded by the patients' wellbeing and tolerance to chemoradiotherapy, subjecting the population to selection bias.

\section{Critiques}

The groundbreaking results of the PACIFIC study represented the first modern improvement in the treatment of stage III NSCLC. At the outset, given the remarkable improvement in PFS of 16.8 months compared to 5.6 months reported from the interim analysis, the outcomes were accepted with enthusiasm. However, there were several criticisms of the study, including concerns of poor survival outcomes in the standard of care arm, the unknown utilization of PET-CT at initial staging and the radiotherapy doses.

The poor median PFS in the placebo arm of 5.6 months appeared low, particularly in comparison to the control arms in previous phase III studies demonstrating median PFS of 9.8 and 11.8 months $(5,10)$. This could potentially lead to overstating the benefit of durvalumab. However, it should be noted that randomization occurred following the completion of chemoradiotherapy in the PACIFIC study, in contrast to RTOG 0617 and PROCLAIM, where randomization occurred prior. This would represent a span of two to three months, which would make the median PFS nearly equivalent. In addition, the median OS, 1-year OS and 2-year OS for the standard arms were comparable among the three studies (Table 1).

Given the international and community involvement in this study, there was significant heterogeneity in the work-up and treatment of study patients. One concern noted was the lack of description for PET-CT utilization in the study. Although PET-CT is routine in the initial staging of LA-NSCLC in several countries, its practice is not ubiquitous globally. Low PET-CT utilization could under-stage patients with stage IV cancers, where the use of ICIs has already been demonstrated to show benefits for PFS and OS. Another critique of the study questions the possible inadequate radiotherapy doses used for treatment. Although the majority of patients received between 54 and 66 Gy (92.4\%), there is no description of how many patients received $54 \mathrm{~Gy}$, which is below the established standard of care minimum dose of 60 Gy to maximize local control (31). Reassuringly, as presented at WCLC 2018, the median radiation dose was $63 \mathrm{~Gy}$ for patients who did not have pneumonitis (32). 
Table 1 Progression-free survival and overall survival in phase III studies for stage III, unresectable NSCLC

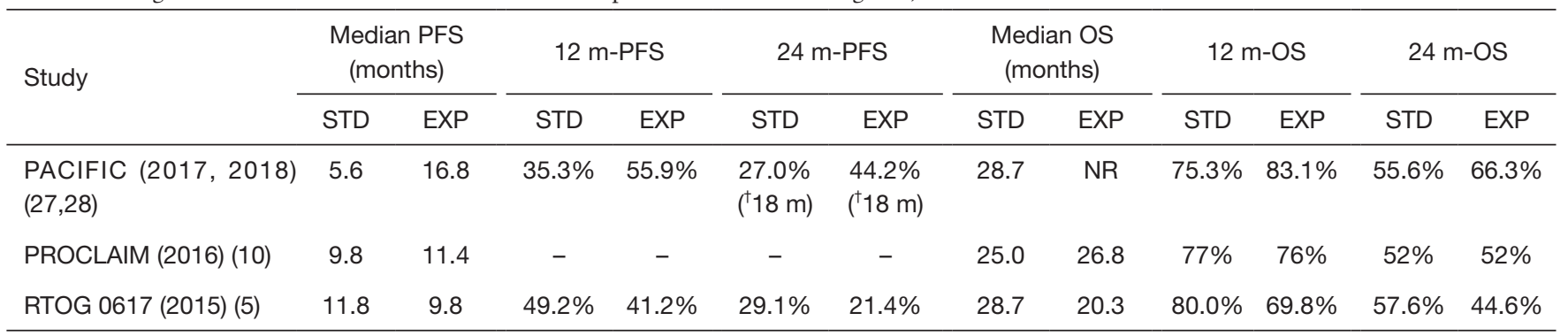

${ }^{\dagger}, 18$ months instead of 24 months. NSCLC, non-small cell lung cancer; PFS, progression-free survival; OS, overall survival; STD, standard arm; EXP, experimental arm; NR, not reached.

\section{Toxicities}

The safety profile of the addition of durvalumab was investigated as a secondary endpoint. Overall, the rates of adverse events were balanced between the two arms. Between durvalumab and placebo, any grade toxicity rates were $96.8 \%$ and $94.9 \%$, while grade 3 or 4 toxicity rates were $30.5 \%$ and $26.1 \%$ (28). This is especially reassuring given the high doses of radiotherapy patients received and concern regarding potential dose-related complications. In the literature, palliative doses of radiotherapy and stereotactic body radiation therapy (SBRT) in combination with PD-1 inhibitors have also shown tolerable rates of immune-related adverse events (33-35).

The frequency of all-grade pneumonitis from any cause was noted to be higher in patients who received consolidative durvalumab with a rate of $32.8 \%$ as compared to $23.5 \%$ in the placebo arm (28). Reassuringly, the rates of grade 3 or 4 pneumonitis were low in both groups: $3.4 \%$ and $2.1 \%(28)$. However, there is no description of rates of grade 2 pneumonitis, a clinically relevant adverse event which often requires close follow-up and treatment with high dose steroids. This could be seen as a limitation of the study and would require more study with clinical practice.

Pneumonitis is a toxicity of special interest as there have been several reports of increased pneumonitis with combination therapy in the literature. For example, a case report described two cases of radiation recall pneumonitis, which reaffirmed that radiotherapy and immunotherapy may be associated with unique risks in addition to benefits (36). Additionally, in a secondary analysis of KEYNOTE-001, the investigators found a significantly increased rate of pneumonitis for patients who received any thoracic radiotherapy prior to receipt of pembrolizumab (13\% vs. $1 \%, \mathrm{P}=0.046)$ (37). To investigate this further in the PACIFIC study, Vansteenkiste et al. presented a sub- analysis of the patients who developed pneumonitis to better characterize the pattern of toxicity. The time to onset and duration of pneumonitis was similar between both arms (durvalumab vs. placebo: 55 vs. 55 days and 64 vs. 57 days) (32). There also did not appear to be a radiation dose-related effect, but prior induction chemotherapy was associated with a lower incidence of pneumonitis in both arms (32). Finally, on multivariate analysis, it was found that patients accrued in Asian countries were more likely to develop pneumonitis (odds ratio 5.40; 95\% CI: 3.16-9.43) (32).

\section{Future studies}

With the promising outcomes of the PACIFIC study, there is greater enthusiasm for other studies in the domain of non-metastatic NSCLC. There are several ongoing studies investigating the use of adjuvant and neoadjuvant PD-1 or PD-L1 inhibitors for resectable NSCLC. For unresectable locally advanced NSCLC, RTOG 3505 was a planned randomized phase III trial investigating the use of consolidative nivolumab compared to placebo in a similar design to the PACIFIC study, but has since been terminated. The PACIFIC-2 study is a randomized phase III study designed to investigate the use of concurrent durvalumab with chemoradiotherapy in addition to consolidation therapy, and is currently accruing (NCT03519971). The use of concurrent therapy is particularly interesting given the favorable outcomes in the subgroup analysis when durvalumab was given closer to the end of chemoradiation. Additionally, Table 2 summarizes other active studies for unresectable stage III NSCLC.

\section{Conclusions}

The treatment paradigm of stage III NSCLC appeared 
Table 2 Active studies with immune checkpoint inhibitors for stage III, unresectable NSCLC

\begin{tabular}{|c|c|c|c|c|c|c|}
\hline Drug & Phase & Enrollment & Sponsor & Primary endpoint(s) & Timing of immunotherapy & $\begin{array}{c}\text { ClinicalTrials.gov } \\
\text { identifier }\end{array}$ \\
\hline \multicolumn{7}{|l|}{ PD-L1 inhibitors } \\
\hline Atezolizumab & II & 52 & M.D. Anderson & $\begin{array}{c}\text { Grade 3-4 non- } \\
\text { hematologic toxicities }\end{array}$ & Consolidation & NCT02525757 \\
\hline \multicolumn{7}{|l|}{ PD-1 inhibitors } \\
\hline Nivolumab & II & 78 & $\begin{array}{l}\text { European Thoracic } \\
\text { Oncology Platform }\end{array}$ & Grade 3+ pneumonitis & Concurrent, consolidative & NCT02434081 \\
\hline Pembrolizumab & I & 30 & Rutgers & MTD, DLT & $\begin{array}{l}\text { Various: 2-6 weeks post- } \\
\text { CRT OR } 2 \text { weeks before } \\
\text { end of CRT OR start of CRT }\end{array}$ & NCT02621398 \\
\hline
\end{tabular}

NSCLC, non-small cell lung cancer; PD-L1, programmed death-ligand 1; PFS, progression-free survival; OS, overall survival; PD-1, programmed cell death protein 1; MTD, maximum tolerated dose; DLT, dose limiting toxicity; CRT, chemo/radiotherapy.

to have plateaued with concurrent chemoradiotherapy. Despite efforts to modify radiotherapy, chemotherapy and even surgical options, outcomes remained poor in this patient population. In this landmark phase III trial, the PACIFIC study demonstrated the efficacy of consolidative immunotherapy in improving survival. Patients who received consolidative durvalumab had higher rates of OS, PFS, distant metastasis free survival and objective response rates, with minimal increases in toxicities. Patients who received durvalumab had higher all-grade pneumonitis rates, but reassuringly, this difference did not extend to higher grade pneumonitis. There were several criticisms of the study including the heterogeneity of work-up and treatment parameters. These limitations may be addressed in the next study, PACIFIC-2, which will investigate the use of adding concurrent durvalumab to consolidative therapy, which has been approved internationally as the standard of care for the treatment of unresectable stage III NSCLC with the publication of PACIFIC.

\section{Acknowledgments}

None.

\section{Footnote}

Conflicts of Interest: JD Schoenfeld reports research funding from Merck and BMS and consulting for BMS, AZ, Nanobiotix, Debiopharm, Tilos, LEK and Catenion. AY Sun reports Speakers Bureau and Advisory Board for AZ. A Bang has no conflicts of interest to declare.

Ethical Statement: The authors are accountable for all aspects of the work in ensuring that questions related to the accuracy or integrity of any part of the work are appropriately investigated and resolved.

\section{References}

1. Bray F, Ferlay J, Soerjamatarm I, et al. Global cancer statistics 2018: GLOBOCAN estimates of incidence and mortality worldwide for 36 cancers in 185 countries. CA Cancer J Clin 2018; 68:394-424.

2. Aupérin A, Le Pechoux C, Rolland E, et al. Meta-analysis of concomitant versus sequential radiochemotherapy in locally advanced non-small-cell lung cancer. J Clin Oncol 2010; 28:2181-90.

3. Bradley JD, Hu C, Komaki RU, et al. Long-Term Results 
of RTOG 0617: A Randomized Phase 3 Comparison of Standard Dose Versus High Dose Conformal Chemoradiation Therapy +/- Cetuximab for Stage III NSCLC. Int J Radiat Oncol Biol Phys 2017;99:S105.

4. Veenstra CM, Vachani A, Ciunci CA, et al. Trends in the Use of 18F-Fluorodeoxyglucose PET Imaging in Surveillance of Non-Small-Cell Lung and Colorectal Cancer. J Am Coll Radiol 2016;13:491-6.

5. Bradley JD, Paulus R, Komaki R, et al. Standarddose versus high-dose conformal radiotherapy with concurrent and consolidation carboplatin plus paclitaxel with or without cetuximab for patients with stage IIIA or IIIB non-small-cell lung cancer (RTOG 0617): a randomized, two-by-two factorial phase 3 study. Lancet Oncol 2015;16:187-99.

6. Speirs CK, DeWees TA, Rehman S, et al. Heart dose is an independent dosimetric predictor of overall survival in locally advanced non-small cell lung cancer. J Thorac Oncol 2017;12:293-301.

7. Raman S, Bissonnette JP, Warner A, et al. Rationale and Protocol for a Canadian multicenter Phase II randomized trial assessing Selective Metabolically Adaptive Radiation Dose Escalation in Locally Advanced Non-Small Cell Lung Cancer (NCT02788461). Clin Lung Cancer 2018;19:e699-e703.

8. Kong FM, Haken RKT, Schipper M, et al. Effect of Midtreatment PET/CT-Adapted Radiation Therapy With Concurrent Chemotherapy in Patients With Locally Advanced Non-Small-Cell Lung Cancer: A Phase 2 Clinical Trial. JAMA Oncol 2017;3:1358-65.

9. Ahn JS, Ahn YC, Kim JH, et al. Multinational Randomized Phase III Trial With or Without Consolidation Chemotherapy Using Docetaxel and Cisplatin After Concurrent Chemoradiation in Inoperable Stage III NonSmall-Cell Lung Cancer: KCSG-LU05-04. J Clin Oncol 2015;33:2660-6.

10. Senan S, Brade A, Wang LH, et al. PROCLAIM: Randomized Phase III Trial of Pemetrexed-Cisplatin or Etoposide-Cisplatin Plus Thoracic Radiation Therapy Followed by Consolidation Chemotherapy in Locally Advanced Nonsquamous Non-Small-Cell Lung Cancer. J Clin Oncol 2016;34:953-62.

11. Borghaei H, Paz-Ares L, Horn L, et al. Nivolumab versus docetaxel in advanced nonsquamous non-small-cell lung cancer. N Engl J Med 2015;373:1627-39.

12. Brahmer J, Reckamp KL, Baas P, et al. Nivolumab versus docetaxel in advanced squamous-cell non-small-cell lung cancer. N Engl J Med 2015;373:123-35.
13. Herbst RS, Baas P, Kim DW, et al. Pembrolizumab versus docetaxel for previously treated, PD-L1-positive, advanced non-small-cell lung cancer (KEYNOTE-010): a randomised controlled trial. Lancet 2016;387:1540-50.

14. Reck M, Rodriguez-Abreu D, Robinson AG, et al. Pembrolizumab versus chemotherapy for PD-L1positive non-small-cell lung cancer. N Engl J Med 2016;375:1823-33.

15. Fehrenbacher L, Spira A, Ballinger M, et al. Atezolizumab versus docetaxel for patients with previously treated non-small-cell lung cancer (POPLAR): a multicentre, open-label, phase 2 randomised controlled trial. Lancet 2016;387:1837-46.

16. Rittmeyer A, Barlesi F, Waterkamp D, et al. Atezolizumab versus docetaxel in patients with previously treated non-small-cell lung cancer (OAK): a phase 3, openlabel, multicentre randomised controlled trial. Lancet 2017;389:255-65.

17. Hellmann MD, Ciuleanu TE, Pluzanski A, et al. Nivolumab plus ipilimumab in lung cancer with a high tumor mutational burden. N Engl J Med 2018;378:2093-104.

18. Gandhi L, Rodriguez-Abreu D, Gadgeel S, et al. Pembrolizumab plus chemotherapy in metastatic nonsmall-cell lung cancer. N Engl J Med 2018;378:2078-92.

19. Lee Y, Auh SL, Wang Y, et al. Therapeutic effects of ablative radiation on local tumor require CD8+ T cells: changing strategies for cancer treatment. Blood 2009;114:589-95.

20. Formenti, SC \& Demaria S. Combining Radiotherapy and Cancer Immunotherapy: A Paradigm Shift. J Natl Cancer Inst 2013;105:256-65.

21. Wara WM. Immunosuppression associated with radiation therapy. Int J Radiat Oncol Biol Phys 1977;2:593-6.

22. Twyman-Saint Victor C, Rech AJ, Maity A, et al. Radiation and dual checkpoint blockade activate non-redundant immune mechanisms in cancer. Nature 2015;520:373-7.

23. Dovedi SJ, Adlard AL, Lipowska-Bhalla G, et al. Acquired resistance to fractionated radiotherapy can be overcome by concurrent PD-L1 blockade. Cancer Res 2014;74:5458-68.

24. Sharabi AB, Tran PT, Lim M, et al. Stereotactic Radiation Therapy Combined With Immunotherapy: Augmenting the Role of Radiation in Local and Systemic Treatment. Oncology (Williston Park) 2015;29:331-40.

25. Stewart R, Morrow M, Hammond SA, et al. Identification and characterization of MEDI4736, an antagonistic antiPD-L1 monoclonal antibody. Cancer Immunol Res 
2015;3:1052-62.

26. Garassino MC, Cho BC, Kim JH, et al. Durvalumab as third-line or later treatment for advanced non-small-cell lung cancer (ATLANTIC): an open-label, single-arm, phase 2 study. Lancet Oncol 2018;19:521-36.

27. Antonia SJ, Villegas A, Daniel D, et al. Durvalumab after chemoradiotherapy in stage III non-small-cell lung cancer. N Engl J Med 2017;377:1919-29.

28. Antonia SJ, Villegas A, Daniel D, et al. Overall survival with durvalumab after chemoradiotherapy in stage III NSCLC. N Engl J Med 2018;379:2342-50.

29. Brookes ST, Whitley E, Egger M, et al. Subgroup analyses in randomized trials: risks of subgroup-specific analyses;: power and sample size for the interaction test. J Clin Epidemiol 2004;57:229-36.

30. Wallis CJD, Butaney M, Satkunasivam R, et al. Association of patient sex with efficacy of immune checkpoint inhibitors and overall survival in advanced cancers: a systematic review and meta-analysis. JAMA Oncol 2019;5:529-36.

31. Perez CA, Stanly K, Grundy G, et al. Impact of irradiation technique and tumor extent in tumor control and survival of patients with unresectable non-oat cell carcinoma of the lung: report by the Radiation Therapy Oncology Group. Cancer 1982;50:1091-9.

32. Vansteenkiste JF, Naidoo J, Faivre-Finn C, et al. MA05.02
PACIFIC subgroup analysis: pneumonitis in stage III, unresectable NSCLC patients treated with durvalumab vs. placebo after CRT. J Thorac Oncol 2018;13:S370-1.

33. Bang A, Wilhite TJ, Pike LRG, et al. Multicenter evaluation of the tolerability of combined treatment with PD-1 and CTLA-4 immune checkpoint inhibitors and palliative radiation therapy. Int J Radiat Oncol Biol Phys 2017;98:344-51.

34. Theelen W, Peulen H, Lalezari F, et al. Randomized phase II study of pembrolizumab after stereotactic body radiotherapy (SBRT) versus pembrolizumab alone in patients with advanced non-small cell lung cancer: The PEMBRO-RT study. J Clin Oncol 2018;36;abstr 9023.

35. Luke JJ, Lemons JM, Karrison TG, et al. Safety and Clinical Activity of Pembrolizumab and Multisite Stereotactic Body Radiotherapy in Patients With Advanced Solid Tumors. J Clin Oncol 2018;36:1611-18.

36. Shibaki R, Akamatsu H, Fujimoto M, Koh Y \& Yamamoto N. Nivolumab induced radiation recall pneumonitis after two years of radiotherapy. Ann Oncol 2017;28:1404-5.

37. Shaverdian N, Lisberg AE, Bornazyan K, et al. Previous radiotherapy and the clinical activity and toxicity of pembrolizumab in the treatment of non-small-cell lung cancer: a secondary analysis of the KEYNOTE-001 phase 1 trial. Lancet Oncol 2017;18:895-903.
Cite this article as: Bang A, Schoenfeld JD, Sun AY. PACIFIC: shifting tides in the treatment of locally advanced non-small cell lung cancer. Transl Lung Cancer Res 2019;8(Suppl 2):S139S146. doi: 10.21037/tlcr.2019.09.04 